Review began 08/24/2021 Review ended 09/22/2021 Published 09/25/2021

\section{() Copyright 2021}

Yang et al. This is an open access article distributed under the terms of the Creative Commons Attribution License CC-BY 4.0. which permits unrestricted use, distribution, and reproduction in any medium, provided the original author and source are credited.

\title{
In Every Man, There Is a Child: Henoch-Schönlein Purpura in an Adult With Liver Cirrhosis
}

\author{
Jiajia Yang ${ }^{1}$, Andrew Okpe ${ }^{2}$, Amogh Pathak ${ }^{2}$ \\ 1. Molecular Pharmacology and Physiology, University of South Florida Morsani College of Medicine, Tampa, USA 2. \\ Internal Medicine, St. Peter's University Hospital, New Brunswick, USA
}

Corresponding author: Amogh Pathak, apathak@saintpetersuh.com

\begin{abstract}
Henoch-Schönlein purpura (HSP), also known as immunoglobulin A (IgA) vasculitis, is a small-vessel vasculitis characterized by IgA deposits in various organs in the body producing a unique constellation of symptoms. This disease predominantly affects the skin (palpable purpura), joints (arthritis/arthralgia), gut (abdominal pain), and kidneys (nephritic syndrome-IgA nephropathy [IgAN]). The pathogenesis of HSP in children is usually secondary to an immune reaction after viral infections. In adults, few cases of HSP/IgA vasculitis have been reported secondary to altered metabolism of IgA in patients with alcoholic liver cirrhosis.
\end{abstract}

Here, we report an unusual case of HSP/IgA vasculitis. The patient presented with signs of alcoholic liver cirrhosis with abdominal pain and ascites along with a lower extremity purpuric rash. The patient had significant findings of liver cirrhosis with radiographic evidence of cirrhotic liver with esophageal varices and splenorenal shunt and elevated serum ascites albumin gradient. Urinalysis revealed proteinuria with microscopic hematuria, further evaluated with a kidney biopsy. Microscopic analysis revealed focal segmental endocapillary and extracapillary proliferative glomerulonephritis with focal necrotizing features, consistent with IgAN/HSP nephritis. Treatment was initiated with high-dose steroids and cyclophosphamide infusions.

Alcohol-induced endotoxin release and inflammation lead to high amounts of circulating IgA due to increased intestinal permeability and reduced hepatic clearance. Further disease development is caused by IgA deposits in affected organs (skin and kidney in our case). We hypothesize that the development of disease for the patient was secondary to altered IgA processing in decompensated alcoholic cirrhosis.

Categories: Internal Medicine, Gastroenterology, Nephrology

Keywords: henoch-schönlein purpura (iga vasculitis), glomerulonephritis, iga nephropathy, liver cirrhosis, alcohol use disorder

\section{Introduction}

Henoch-Schönlein purpura (HSP, also known as immunoglobulin A [IgA] vasculitis) often involves the skin, gastrointestinal tract, kidney, and frequently causes arthritis [1]. HSP is primarily a childhood disease, affecting children between the ages of three and fifteen years, with a male predominance [2]. In adults presenting with HSP, intussusception is rarely reported, but the risk of progression of renal involvement to end-stage kidney disease is higher.

HSP nephritis may appear indistinguishable from immunoglobulin A nephropathy (IgAN) [1]. IgAN is characterized by the presence of IgA dominant or codominant mesangial deposits and is the most prevalent primary glomerulonephritis worldwide. Clinically, IgAN manifests with hematuria/proteinuria and impaired kidney function. It is classified as the primary idiopathic form and the secondary form that is associated with chronic liver disease (the leading secondary cause), chronic infections, and neoplasms [3]. Cirrhosis has been commonly implicated with IgA mesangial deposits with fewer other immunoglobulins and C3 in the glomeruli. The term cirrhotic glomerulonephritis is used to describe this pathological phenomenon [4].

\section{Case Presentation}

A 56-year-old Caucasian male with a history of alcohol use disorder presented to the hospital complaining of abdominal distention and moderately severe nonlocalizing aching pain all over the abdomen for one week and lower extremity petechial rash for four days.

Physical examination showed globular distention of the abdomen (Figure 1) with dullness to percussion in bilateral flanks with shifting dullness. In addition, he had an abdominal wall ventral hernia extending from the xiphisternum to the umbilicus. Examination of the skin also showed flat, nonblanching, pinpoint, round, reddish-brown petechial rash in clusters on bilateral shins (Figure 2) and abdomen (Figure 1). 


\section{Cureus}

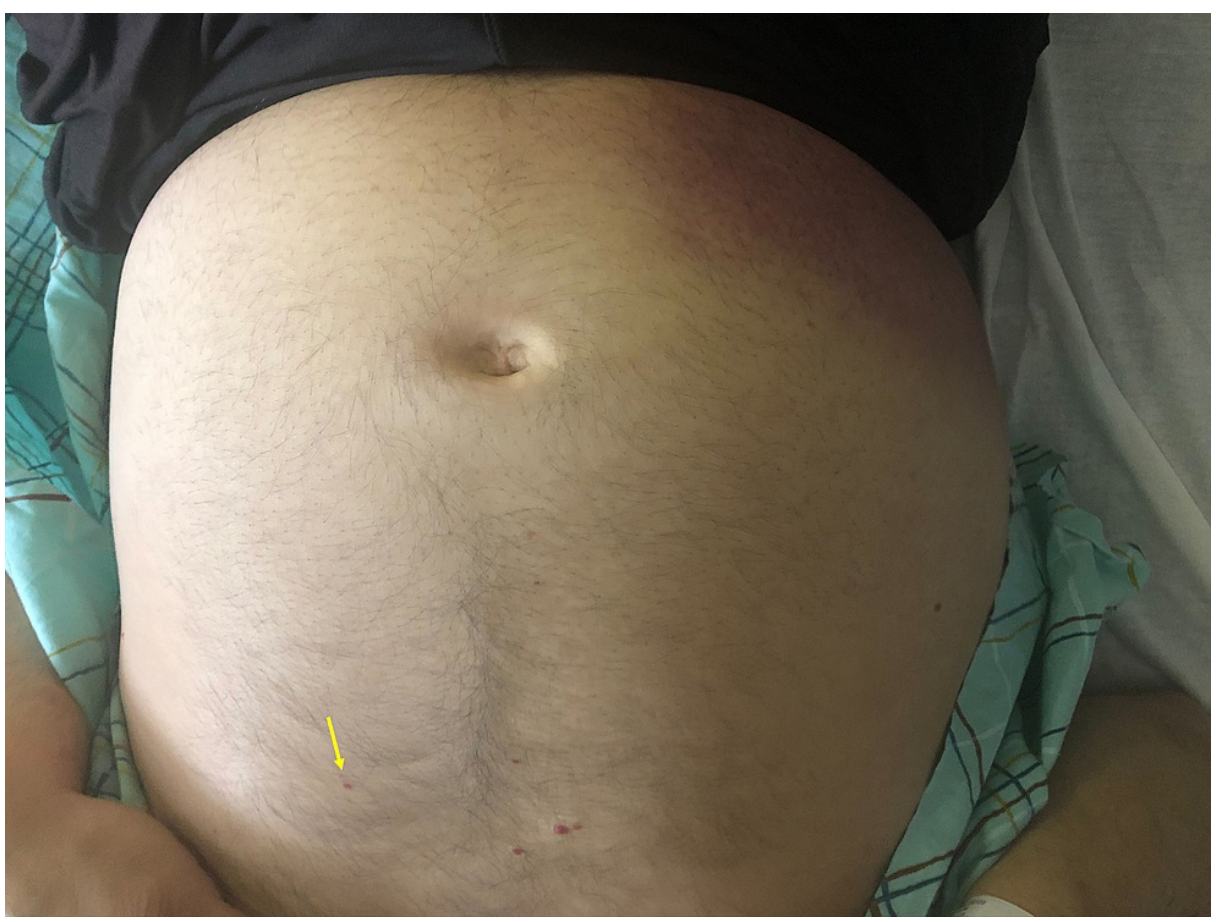

FIGURE 1: Abdominal distension with the petechial rash on the skin.

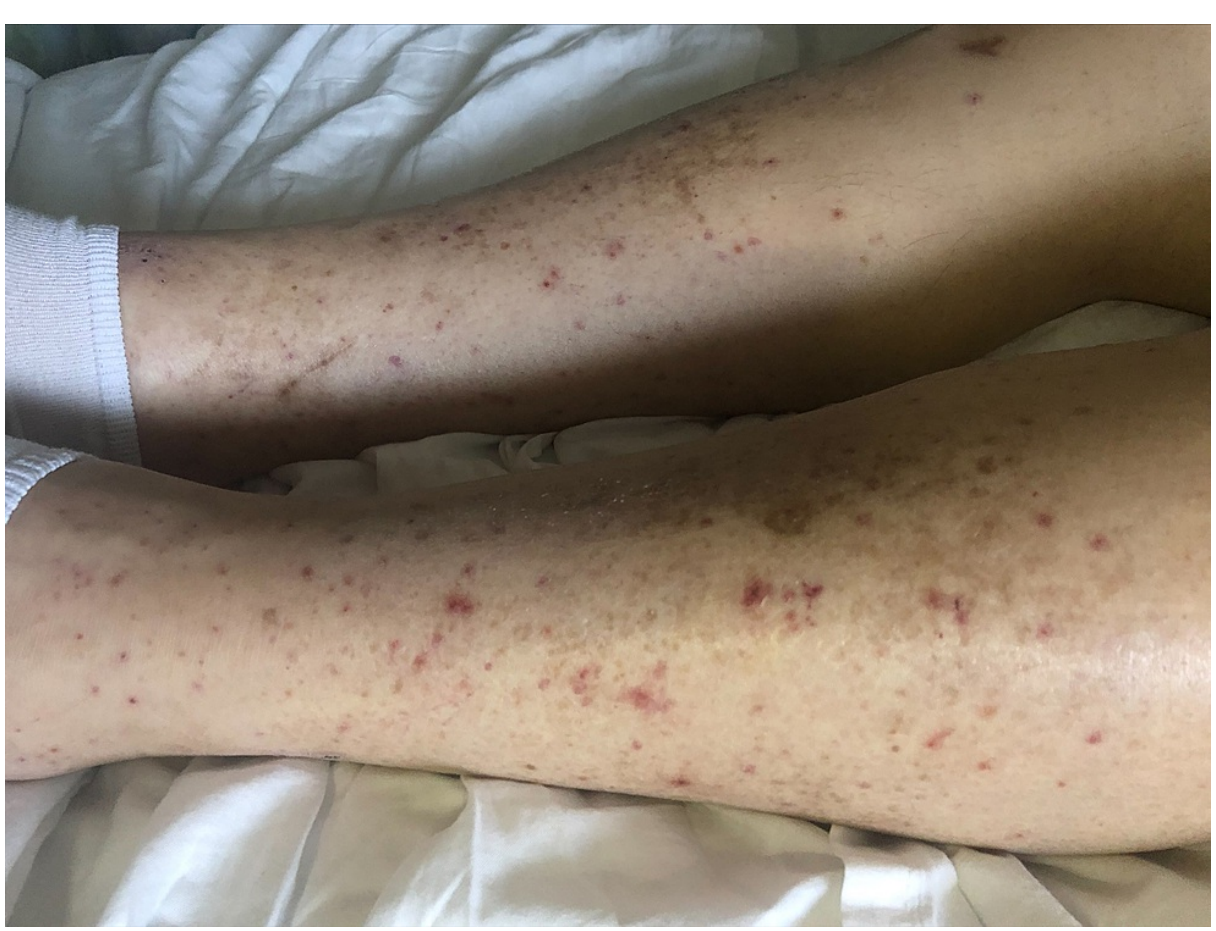

FIGURE 2: Bilateral lower extremity petechial rash.

The patient underwent computed tomography (CT) scan of the abdomen and paracentesis. CT scan revealed cirrhotic liver with ascites, extensive esophageal varices, and splenorenal shunt. Ultrasound further revealed signs of portal hypertension with splenomegaly. Diagnostic and therapeutic paracentesis was performed, with fluid studies nonindicative of spontaneous bacterial peritonitis. The albumin level of ascitic fluid was $0.6 \mathrm{~g} / \mathrm{dL}$, with a serum ascites albumin gradient of $1.9 \mathrm{~g} / \mathrm{dL}$.

Routine complete blood count was significant for hemoglobin of $13.6 \mathrm{~g} / \mathrm{dL}$ with macrocytosis (mean corpuscular volume of $100.9 \mathrm{\mu m}^{3}$ ) and thrombocytopenia $\left(77,000 / \mathrm{mm}^{3}\right)$. Effects of liver cirrhosis were apparent with the international normalized ratio of 1.26 , low albumin $(2.5 \mathrm{~g} / \mathrm{dL})$, and bilirubin of $3.9 \mathrm{mg} / \mathrm{dL}$ 


\section{Cureus}

(with an indirect component of $2.6 \mathrm{mg} / \mathrm{dL}$ ). Liver transaminases were within normal limits, and alkaline phosphatase was elevated at $131 \mathrm{U} / \mathrm{L}$.

The patient had reduced kidney function with a creatinine of $1.61 \mathrm{mg} / \mathrm{dL}$ and calculated glomerular filtration rate of $54 \mathrm{~mL} /$ minute. Urinalysis showed proteinuria $(192 \mathrm{mg} / \mathrm{dL})$, with elevated protein creatinine ratio $(2,526 \mathrm{mg} / \mathrm{g})$, and microscopic hematuria (packed red blood cell $[\mathrm{RBC}]$ ) with no RBC cast. Further biochemical analysis revealed elevated serum iron, iron saturation, and normal ferritin. Serum IgA levels were elevated at more than three times the upper limit.

The patient's kidney function continued to worsen. To further evaluate the etiology and establish the diagnosis for glomerulonephritis, the patient underwent a kidney biopsy. Biopsy was significant for focal segmental endocapillary and extracapillary proliferative glomerulonephritis with focal necrotizing features (Figure 3). Immunofluorescence staining was positive for IgA deposits (Figure 4A), consistent with IgAN. Hematoxylin and eosin staining of kidney biopsy showed degenerating RBC cast (Figure $4 B$ ). Therapy was initiated with high-dose steroids and cyclophosphamide infusions. The clinical course was complicated by an upper gastrointestinal bleed from the rupture of esophageal varices for which the patient needed a transjugular intrahepatic portosystemic shunt. The patient was continued with a further course of cyclophosphamide infusions with monitoring of kidney functions.
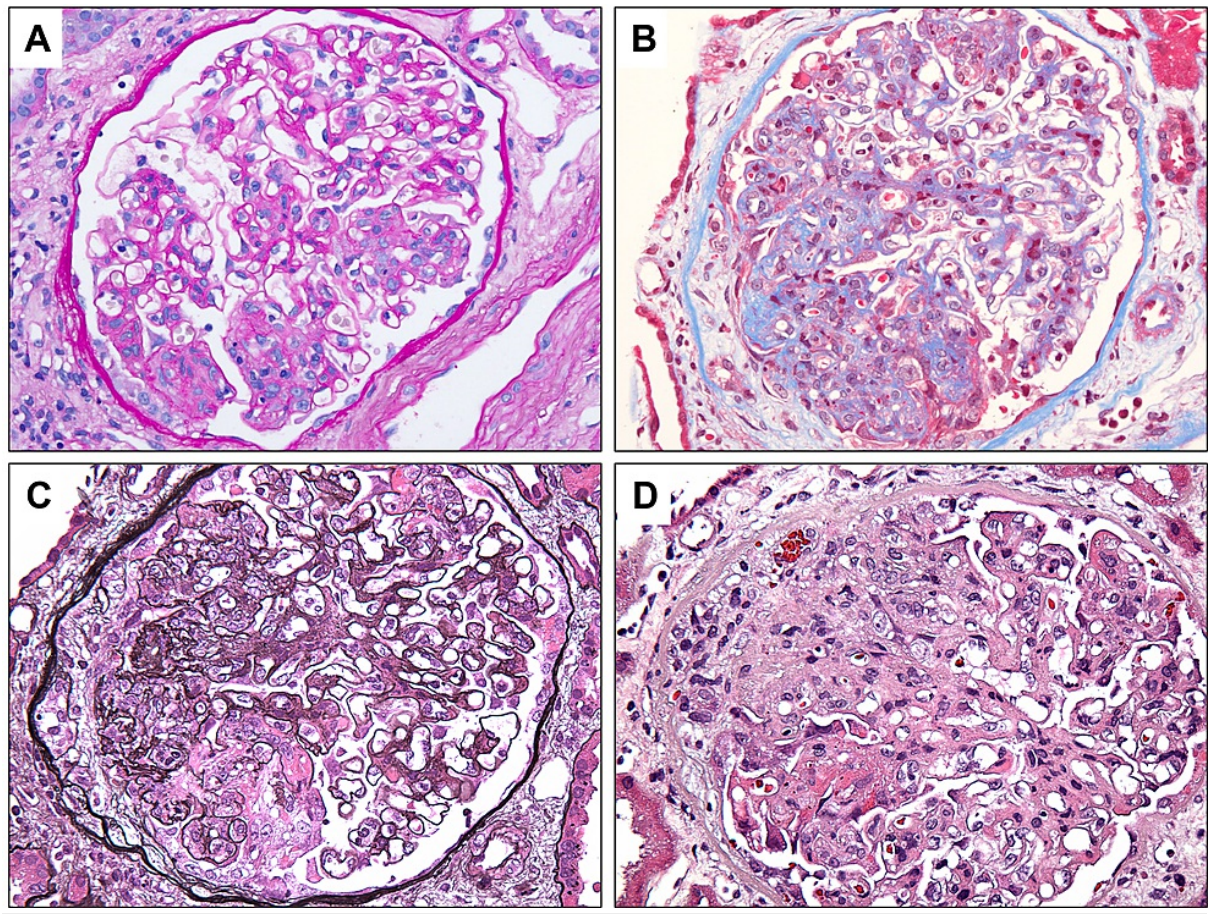

FIGURE 3: Light microscopic observation of kidney biopsy.

Periodic acid-Schiff stain shows glomerulus with segmental endocapillary proliferation (A). Trichrome stain shows glomerulus with fibrocellular crescent (B). Jones' methenamine silver stain shows endocapillary proliferation and cellular crescent (C). Hematoxylin and eosin stain shows fibrinoid necrosis and crescent (D). Original magnification $400 \times$. 


\section{Cureus}
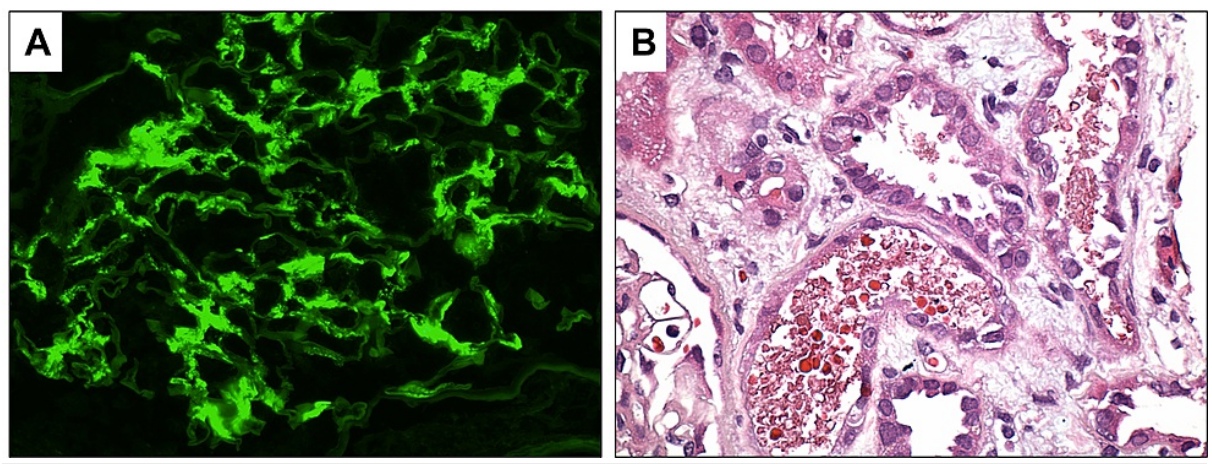

FIGURE 4: Renal biopsy of the patient.

Immunofluorescence reveals mesangial deposits of immunoglobulin A (A). Hematoxylin and eosin stain shows degenerating red blood cell cast (B). Original magnification $400 x$.

\section{Discussion}

In our patient, renal biopsy confirmed IgA deposit accumulation in the kidney likely secondary to alcoholic cirrhosis. Mesangial IgA deposition, which does not necessarily imply IgAN, is a frequent finding in autopsy studies in chronic liver disease [5]. However, we demonstrate an infrequent case in which our patient's presentations including purpura, renal involvement, and abdominal pain met the latest criteria for diagnosing IgA vasculitis/HSP in adults [6]. So far, only 13 cases in addition to the presented case have reported the rare association between liver cirrhosis and HSP $[7,8]$.

Experimentally, the relationship between excessive alcohol intake-associated hepatocellular injury and IgA nephropathy has been validated by rat models. Intense IgA deposition, mild mesangial expansion, and foot process effacement, as well as severe proteinuria, have been observed in these animal models following chronic ethanol ingestion $[9,10]$. Regarding patients diagnosed with cirrhosis, recent evidence also showed 67 biopsies had IgA-containing immune complex deposits within a total cohort of 118 cirrhotic patients [11]. Emerging case reports further illustrate that alcohol abuse possibly contributes to the development of secondary IgA nephropathy [12-14].

Mechanistically, alcohol induces the expression of the microsomal ethanol oxidation system (CYP2E1) and oxidative stress, resulting in increased gastrointestinal permeability and liver inflammation. The downstream effect is IgA overload caused by elevated intestinal production and reduced hepatic immune complex clearance [15].

Overall, $87 \%$ of patients with measurable IgA-containing immune complexes in serum demonstrated IgA skin deposits [16]. Of note, our patient had significantly increased serum IgA. In addition, the purpuric rash seen in our patient is considered to be a sign of IgA vasculitis, a systemic form of IgAN. However, the petechial rash can be caused by thrombocytopenia among cirrhotic patients. A skin biopsy is needed to confirm the presence of IgA deposits in blood vessels of the skin.

The treatment for cirrhosis-related IgAN focuses on the underlying liver condition; in this case, it is important to educate patients on alcohol abstinence. Several traditional immunosuppressive agents such as corticosteroids, cyclophosphamide, azathioprine, and mycophenolate mofetil have been tried to manage IgAN [17]. Furthermore, evidence has shown that the addition of angiotensin-converting enzyme inhibitors (ACEIs) or angiotensin receptor blockers (ARBs) to corticosteroids may be important in preserving proteinuric IgA nephrotic patients' kidney function in the long-term follow-up [18-20].

\section{Conclusions}

We described the case of an uncommon patient who presented with alcoholic liver disease associated with microscopic hematuria, proteinuria, reduced glomerular filtration rate, IgA mesangial deposits, and skin rash, identifying a secondary form of IgA vasculitis/HSP. Even though HSP in adults is rare, it is important for physicians to consider urinalysis and kidney/skin biopsy in the context of liver cirrhosis and rash, and for patients to rigorously abstain from alcohol to slow down the progression of the disease. Multiple clinical trials have provided valuable data on the benefits of kidney function regeneration with the treatment of ACEIs/ARBs.

\section{Additional Information \\ Disclosures}


Human subjects: Consent was obtained or waived by all participants in this study. Conflicts of interest: In compliance with the ICMJE uniform disclosure form, all authors declare the following: Payment/services info: All authors have declared that no financial support was received from any organization for the submitted work. Financial relationships: All authors have declared that they have no financial relationships at present or within the previous three years with any organizations that might have an interest in the submitted work. Other relationships: All authors have declared that there are no other relationships or activities that could appear to have influenced the submitted work.

\section{References}

1. Jennette JC, Falk RJ, Bacon PA, et al.: 2012 revised International Chapel Hill Consensus Conference Nomenclature of Vasculitides. Arthritis Rheum. 2013, 65:1-11. 10.1002/art.37715

2. Piram M, Mahr A: Epidemiology of immunoglobulin A vasculitis (Henoch-Schönlein): current state of knowledge. Curr Opin Rheumatol. 2013, 25:171-8. 10.1097/BOR.0b013e32835d8e2a

3. Saha MK, Julian BA, Novak J, Rizk DV: Secondary IgA nephropathy. Kidney Int. 2018, 94:674-81 10.1016/j.kint.2018.02.030

4. Fisher ER, Perez-Stable E: Cirrhotic (hepatic) lobular glomerulonephritis. Correlation of ultrastructural and clinical features. Am J Pathol. 1968, 52:869-89.

5. Johnson RJ, Feehally J Floege J, Tonelli M: Comprehensive clinical nephrology. Elsevier, Edinburgh, UK; 2019.

6. Ozen S, Pistorio A, Iusan SM, et al.: EULAR/PRINTO/PRES criteria for Henoch-Schönlein purpura, childhood polyarteritis nodosa, childhood Wegener granulomatosis and childhood Takayasu arteritis: Ankara 2008. Part II: final classification criteria. Ann Rheum Dis. 2010, 69:798-806. 10.1136/ard.2009.116657

7. Gupta N, Kim J, Njei B: Spontaneous bacterial peritonitis and Henoch-Schönlein purpura in a patient with liver cirrhosis. Case Rep Med. 2015, 2015:340894. 10.1155/2015/340894

8. Bommareddy K, Alreshq R, Jones D, Singh G: A very rare case of immunoglobulin A vasculitis in an adult with alcoholic liver cirrhosis. J Dermatol Skin Sci. 2020, 2:9-12.

9. Smith SM, Yu GS, Tsukamoto H: IgA nephropathy in alcohol abuse. An animal model . Lab Invest. 1990, 62:179-84.

10. Amore A, Coppo R, Roccatello D, et al.: Experimental IgA nephropathy secondary to hepatocellular injury induced by dietary deficiencies and heavy alcohol intake. Lab Invest. 1994, 70:68-77.

11. Hemminger J, Arole V, Ayoub I, Brodsky SV, Nadasdy T, Satoskar AA: Acute glomerulonephritis with large confluent IgA-dominant deposits associated with liver cirrhosis. PLoS One. 2018, 13:e0193274. 10.1371/journal.pone.0193274

12. Suga T, Endoh M, Miura M, Tomino Y, Nomoto Y, Sakai H: Hepatic glomerulosclerosis with IgA deposition. Report of three cases. Tokai J Exp Clin Med. 1983, 8:317-24.

13. Takada D, Sumida K, Sekine A, et al.: IgA nephropathy featuring massive wire loop-like deposits in two patients with alcoholic cirrhosis. BMC Nephrol. 2017, 18:362. 10.1186/s12882-017-0769-1

14. Denha E, Rahim A, Modi S, Oyibo O, Scott M: A 55-year-old male presenting with a lower extremity rash: a case of immunoglobulin A (IgA) nephropathy. Cureus. 2021, 13:e14165. 10.7759/cureus.14165

15. Varga ZV, Matyas C, Paloczi J, Pacher P: Alcohol misuse and kidney injury: epidemiological evidence and potential mechanisms. Alcohol Res. 2017, 38:283-8.

16. van de Wiel A, Valentijn RM, Schuurman HJ, Daha MR, Hené RJ, Kater L: Circulating IgA immune complexes and skin IgA deposits in liver disease. Relation to liver histopathology. Dig Dis Sci. 1988, 33:679-84. 10.1007/BF01540430

17. Selvaskandan H, Cheung CK, Muto M, Barratt J: New strategies and perspectives on managing IgA nephropathy. Clin Exp Nephrol. 2019, 23:577-88. 10.1007/s10157-019-01700-1

18. Praga M, Gutiérrez E, González E, Morales E, Hernández E: Treatment of IgA nephropathy with ACE inhibitors: a randomized and controlled trial. J Am Soc Nephrol. 2003, 14:1578-83. 10.1097/01.asn.0000068460.37369.dc

19. Coppo R, Peruzzi L, Amore A, et al.: IgACE: a placebo-controlled, randomized trial of angiotensinconverting enzyme inhibitors in children and young people with IgA nephropathy and moderate proteinuria. J Am Soc Nephrol. 2007, 18:1880-8. 10.1681/ASN.2006040347

20. Manno C, Torres DD, Rossini M, Pesce F, Schena FP: Randomized controlled clinical trial of corticosteroids plus ACE-inhibitors with long-term follow-up in proteinuric IgA nephropathy. Nephrol Dial Transplant. 2009, 24:3694-701. 10.1093/ndt/gfp356 